\title{
Anlotinib Potentially Eliminates Leukemia Stem Cells and Modulates the Chemosensitivity via Inhibiting JAK2-STAT3/5 Signaling
}

\section{Yuelong Jiang}

The First Affiliated Hospital of Xiamen University

Long 䒽

The First Affiliated Hospital of Xiamen University https://orcid.org/0000-0001-9528-0241

\section{Zhifeng Li}

The First Affiliated Hospital of Xiamen University

\section{Liying Feng}

The First Affiliated Hospital of Xiamen University

\section{Zhijuan Lin}

The First Affiliated Hospital of Xiamen University

\section{Qiuling Chen}

The First Affiliated Hospital of Xiamen University

\section{Guoshu Chen}

Central Hospital: Hopital Central

Jixiang He

Dongguan People's Hospital

\section{Guowei Li}

Central Hospital: Hopital Central

\section{Yirong Jiang}

Dongguan People's Hospital

Bing Xu ( $\nabla$ xubing@xmu.edu.cn)

The First Affiliated Hospital of Xiamen University https://orcid.org/0000-0002-7271-4438

\section{Research Article}

Keywords: acute myeloid leukemia, leukemia stem cell, anlotinib, JAK, STAT3, chemoresistance

Posted Date: June 7th, 2021

DOI: https://doi.org/10.21203/rs.3.rs-562151/v1 
License: (c) (i) This work is licensed under a Creative Commons Attribution 4.0 International License. Read Full License 


\section{Abstract}

Leukemia stem cells (LSCs) remain as the critical barrier to cure of acute myeloid leukemia (AML) due to its chemoresistance. Here, we explore the role anlotinib -a multiple tyrosine kinases inhibitor in killing LSCs and regulating the chemoresistance. We found anlotinib could effectively induce apoptosis of LSClike cells as well as primary CD34+ AML LSCs while sparing the normal mononuclear cells in vitro. The anti-leukemia activity of anlotinib was also confirmed in the mice model with Kasumi-1 cells; we further found that anlotinib could impair the regeneration capacity of LSCs in the patient-derived leukemia xenograft mouse model. Mechanistically, anlotinib could not only inhibit phosphorylation of c-kit and JAK2 /STAT3 and STAT5 but also downregulate STAT3 and STAT 5 expression. In addition, anlotinib downregulated the anti-apoptotic protein $\mathrm{Bcl}-2$ and $\mathrm{Bcl}-\mathrm{xl}$ and upregulated Bax, thereby enhancing the sensitivity of LSCs to idarubicin in vitro. In conclusion, our results demonstrated anlotinib showed antiLSCs activity and enhanced the chemosensitivity via inhibiting JAK2/STAT signaling in preclinical study and provided a rational basis for combinatory strategies that invoving anlotinib and idarubicin.

\section{Introduction}

The treatment of acute myeloid leukemia $(\mathrm{AML})$ has been rapidly evolved from conventional chemotherapy to target therapeutics such as FLT3-ITDIIDH2 inhibitors and subsequently contribute to an improvement in complete remission rates over the past decade ${ }^{10}$. However, novel therapeutics did not significantly reduce the relapse of $A M L$, which led to the failure to converting complete remission to long term survival of patients ${ }^{14}$. Consequently, preventing relapse remains to be the key point during the management of AML.

Leukemia stem cells are a small population at the ape of AML differentiation hierarchy and characterized by its self-renewal and chemoresistance property ${ }^{16}$. LSCs are considered as the source of relapse after chemotherapy ${ }^{6,17}$. Therefore, targeting LSC is a rationally promising strategy to prevent AML relapse. As previously reported, deprivation of cytokines such IL-3, G-CSF, SCF could induce gradual loss of stemness of LSC ${ }^{16}$. Accordingly, the phenotypic features such IL-3 receptor (CD123) and IL-2 receptor (CD25) high expression confer its capacity to receive cytokines signaling $4,9,11$. Recently, et al reported LSC displayed high expression of multiple growth factor receptors such c-kit, FMS-related tyrosine kinase 3(FLT3), which incurred the overactivation of their downstream tyrosine kinases such as Janus kinase 2 (JAK2) ${ }^{4}$. The JAK family of nonreceptor tyrosine kinases are critical mediators of cytokines and growth factor signaling ${ }^{12}$ and represent an important signaling mechanism supporting AML LSC growth and survival ${ }^{2}$. JAK/STAT signaling could also regulate anti-apoptotic protein such as $\mathrm{Bcl}-2$ and $\mathrm{BCl}-\mathrm{xl}$ as well as pro-apoptotic proteins ${ }^{18}$. Inhibition of JAK2 signaling could contribute to the blockade of LSC proliferation ${ }^{4}$, which implicated that targeting growth factor receptors and their downstream signaling might be effective to eliminate LSC. However, most tyrosine kinases inhibitor displayed their specificity on the target, which might not be suitable in multiple tyrosine kinase activation settings of LSC rationally. 
Anlotinib as multiple kinase inhibitor could target c-kit, platelet derived growth factor receptor (PDGF-R), fibroblast growth factor receptor (FGF-R), vascular endothelial growth factor receptor (VEGF-R) has displayed its efficacy in non-small cell lung cancer and other cancers with mild adverse complications ${ }^{13}$. Additionally, Liang $L$ et al reported anlotinib could exert its effect by inhibiting JAK2 signaling ${ }^{7}$. Our previous data showed that other tyrosine inhibitor such as apatinib displayed its efficacy in elimination of AML cells ${ }^{5}$. Therefore, our study aim to exploring the effect of anlotinib in elimination of LSC and its potential mechanism.

\section{Materials And Methods}

\section{Cell line culture}

Kasumi-1 and KG-1a cell lines were stored in our own laboratory.Kasumi-1 was cultured in RPMI-1640 (HyCloneTM, Logan, UT, USA) supplemented with $100 \mathrm{U} / \mathrm{ml}$ penicillin and $100 \mu \mathrm{g} / \mathrm{ml}$ streptomycin $(1 \times$ $\mathrm{P} / \mathrm{S}$ ) and $10 \%$ fetal bovine serum (FBS) (Hy Clone TM, Logan, UT, USA).KG-1 a was cultured in IMDM (HyClone TM, Logan, UT, USA) supplemented with $100 \mathrm{U} / \mathrm{ml}$ penicillin and $100 \mu \mathrm{g} / \mathrm{ml}$ streptomycin $(1 \times$ $\mathrm{P} / \mathrm{S}$ ) and $10 \%$ fetal bovine serum (FBS) (Hy Clone TM, Logan, UT, USA).

\section{Patients and donor samples}

Primary samples from bone marrow of AML patients $(n=9)$ and peripheral blood of healthy donors $(n=$ 8) were collected in Department of Hematology, First Affiliated Hospital of Xiamen University after patients were informed consent. This study was carried out in accordance with the Declaration of Helsinki and approved by the Ethics Review Board of First Affiliated Hospital of Xiamen University, Clinical characteristics of patients with AML are summarized in Table 1. Mononuclear cells were isolated by density gradient centrifugation using Ficol (BD, Franklin Lakes, NJ, USA) and supplemented with RPMI1640 and 10\% FBS for short culture.

\section{Cell viability assay}

Cell proliferation was determined by a CCK-8 kit (MCE, Shanghai, China). Briefly, cells $\left(3 \times 10^{4} \mathrm{cells} /\right.$ well) were plated into 96 -well plates containing $100 \mu \mathrm{l}$ of growth medium and then treated with designated doses of anlotinib for $48 \mathrm{~h}$ and $72 \mathrm{~h}$. CCK-8 agent was added and incubated for 2-4 $\mathrm{h}$ in an incubator after treatment and the absorbance at $450 \mathrm{~nm}$ were read by a VERSA max microplate reader (Molecular Devices, Sunnyvale, CA, USA). All experiments were repeated three times and performed in triplicate in each experiment.

\section{Flow cytometry detection of apoptosis}

Apoptosis of LSC or LSC-like cells was detected by flow cytometry after staining of Annexin V and PI following the manufacturer's instruction. In brief, cells exposed to different treatments were harvested and washed with ice-cold PBS (Gibco, USA) and $1 \times$ Annexin V binding buffer and then subjected to 
Annexin V-FITC/PI staining. Flow cytometry analysis was performed within $1 \mathrm{~h}$ after staining. Primary samples with spontaneous apoptosis over $30 \%$ without treatment were excluded. The specific apoptosis was adopted to adjust the variation in basal levels of spontaneous cell death following the formula: (\% apoptosis in treated cells - \% apoptosis in untreated cells $) /(1-\%$ apoptosis in untreated cells) to analysis of the potential relationship between patients' characteristics and ex vivo efficacy of anlotinib,

\section{Western blot analysis}

Cells with $2 \times 10^{5} /$ well were treated with anlotinib for $12 \mathrm{~h}$. Apoptosis of cells were tested to control apoptotic rates no more than $10 \%$ before immunoblot assays. Hereafter cells mentioned above were lysed in RIPA solution containing protease inhibitor cocktails. The protein level of each sample was determined by a BCA protein Assay (Pierce, Thermo Scientific, USA). protein ( $20 \mu \mathrm{g} /$ lane) was separated by SDS-PAGE, and transferred to a PVDF membrane (Millipore, UK), blocked by $5 \%$ non-fat milk, and incubated with primary antibodies included anti-c-KIT(18691-1-AP, proteintech,USA) and antibodies from Cell Signaling Technology, Danvers, MA, USA including anti-JAK-2(3230), anti-Jak-2 (3230)『anti-PhosphoJak-2(8082) 『anti-Stat3(30835), anti-Stat3(9339S), anti-Phospho-Stat3(4113S), anti-Stat5(94205S), antiPhospho-Stat5(4322S,), anti-Bcl-2(4223S), anti-Bcl-xl(2764S), anti-BAX(5023S), anti- Phospho- c-KIT (3073S), and anti-GAPDH(D16H11). After incubation with HRP-conjugated secondary antibodies, the signals were detected using an enhanced ECL substrate (GE Healthcare, Chicago, USA) and visualized using the Amersham Imager 600 (Al600, GE Healthcare, Chicago, USA).

\section{Mice models}

All animal experiments were approved by the Animal Center of Xiamen University (Xiamen, China). Kasumi- 1 cells $\left(6 \times 10^{6}\right)$ were subcutaneously injected into 6- weeks-old BALB/c male nude mice (Animal Center of Xiamen University, Xiamen, China). The tumor volume $(V)$ was calculated using $V=L * W^{2} / 2(L$ : tumor length, and W: tumor width) twice a week. After tumor volume reached $50 \mathrm{~mm}^{3}$, mice were randomly divided into 2 groups ( $n=5 /$ group) by oral gavage of vehicle or anlotinib $(6 \mathrm{mg} / \mathrm{kg} / \mathrm{d})$ for 1 week respectively. All mice were sacrificed after anlotinib exposure for 7 days.

\section{Human AML xenograft experiments}

Pre-treated bone marrow mononuclear cells of AML patients with anlotinib $(2.5 \mu \mathrm{M})$ or vechicle for $12 \mathrm{~h}$ respectively in vitro. To exclude the impact of apoptotic cells on engraftment of LSCs, we detected apoptosis of the two groups. The equal accounts of alive cells were injected into tail vein of NOD-Prkdc-/IL2rg -/-(NSG) mice (purchased from DMO Itd., Beijing, China) after 1Gy irradiation. In order to confirm the engraftment, the peripheral blood will be stained with human CD45.At the end of this experiment, the NSG mice will be culled to analyze the tumor burden with human CD45-FITC (clone HI30, Biolegend) and murine CD45-BV421 antibodies (clone 30- F11, Biolegend) as well as CD34-APC( clone 581,Biolegend ).

\section{Statistical analysis}


The value was expressed as the mean \pm standard deviation (S.E.M) for at least three independent experiments. The Student t-test or Wilcoxon and Mann-Whitney tests were applied in two-group comparison according to distribution of the data. Multiple-group comparisons were conducted using the One-way analysis of variance (ANOVA) followed by the Bonferroni post hoc test. $P<0.05$ was considered statistically significant. All statistical analyses were performed using SPSS 22.0 software (La Jolla, CA).

\section{Results}

\section{Anlotinib exerted potential anti-LSCs effects by in inhibiting proliferation and inducing apoptosis of LSC- like cells in vitro}

The CD34 ${ }^{+} \mathrm{CD} 38^{-}$cell population in two leukemia stem-like cell lines (LSC-like cells) KG-1a and Kasumi-1 was sorted by magnetic activated cell sorter (MACS), respectively. To evaluate effect of anlotinib on cell proliferation in LSC-like cell lines, we treated CD34+ ${ }^{+}$D $38^{-}$KG-1Aa and $\mathrm{CD} 34^{+} \mathrm{CD} 38^{-}$Kasumi- 1 cells with indicated concentrations of anlotinib for $24 \mathrm{~h} \otimes 48 \mathrm{~h} \otimes 72 \mathrm{~h}$ and measured cell proliferation by CCK-8 assay. The results showed that anlotinib treatment significantly inhibited Kasumi-1 and KG-1 a proliferation in a dose-and time-dependent manner (Fig.1A, B). To investigate whether cell proliferation blockade were caused by apoptosis or cell cell cycle arrest. As a result, significantly higher percentages of apoptosis after anlotinib treatment in a dose- and time dependent manner were observed (Fig.1C, D). In addition, cell cycle arrest in G2/M phase were also observed after anlotinib treatment (Fig.1E, F). Therefore, these data indicated that the anti-LSC potential of anlotinib might be dependent on its cytotoxicity and the capacity of inducing cell cycle arrest in G2/M phase.

\section{Anlotinib targets primary CD34+ AML cells while sparing normal hematopoietic cells ex vivo}

Despite anlotinib could effectively kill LSC-like cells, the exact effect of anlotinib on primary LSCs remains unclear. Therefore, we treated the isolated mononuclear cells from the bone marrow of $9 \mathrm{AML}$ patients with anlotinib with escalating doses. Clinical characteristics of AML patients were summarized in Table 1. Consistent with the anti-leukemia effect in LSC-like cell lines, anlotinib could also significantly induce the apoptosis of CD34+ AML cells in a dose-dependent manner (Fig.2A). In addition, we further evaluated the adverse effects of anlotinib on normal blood cells. Mononuclear cells from the peripheral blood of healthy donors were treated with anlotinib for $48 \mathrm{~h}$. Intriguingly, even the highest dose $(10 \mu \mathrm{M})$ did not show significant cytotoxicity of mononuclear cells from peripheral blood the donors(Fig.2B).

\section{Anlotinib exerted anti-leukemia effects and impaired the regeneration capacity of LSCs in vivo}

To further investigate the anti-LSCs activity in vivo, we establish a subcutaneous leukemia xenograft mice model with Kasumi-1 cell line. Anlotinib was administered after tumor volume reached $50 \mathrm{~mm}^{3}$. As shown in figure, the tumor volume were significantly lower in the group treated by anlotinib after 7 days (Fig.3A). Regeneration of leukemia is the feature of LSCs that distinguish leukemia cells. Whether anlotinib could impair the regeneration capacity of LSCs remains unknown. Mononuclear cells of bone marrow from AML patients were pretreated by anlotinib or vehicle in vitro for $12 \mathrm{~h}$, the same amounts of 
alive cells were injected into tail vein of NSG mice to establish the PDX model of AML. As a result, we found anlotinib pretreatment significantly decrease the engraftment of hCD45+cells and hCD45+CD34+ cells in bone marrow of mice at the $4^{\text {th }}$ week(Fig.3C, D); additionally, the spleen weight in anlotinib group was also significantly less than the vehicle group(Fig.3B). Taken together, anlotinib could not only exert effective anti-leukemia effects but also could suppress the regeneration capacity of LSCs derived from AML patients.

\section{The anti-LSCs activity of anlotinib might be associated with the inhibition of JAK-STAT pathway.}

Overactivation of JAK/STAT signaling is constitutively increased in AML stem and progenitor cells due to the mutations of RTKs or growth factors stimulation and responsible for the self-renewal of LSCs ${ }^{3}$. Whether anlotinib could inhibit JAK-STAT pathway remains to be elucidated. We found that anlotinib could significantly inhibit the activation of c-kit and JAK2. Moreover, the phosphorylation of downstream transcript factors of JAK2 including STAT3 and STAT5 were inhibited after anlotinib treatment. Notably, the expression of STAT3 AND STAT5 were decreased by anlotinib as well. These results indicated anlotinib not only inhibited the activation of JAK2/STAT signaling but also downregulated STAT3 and STAT5 (Fig.4).

\section{Anlotinib enhanced the chemosensitivity of LSCs via regulating apoptotic related-proteins.}

Chemoresistance is considered as the key feature of $\operatorname{LSCs}^{9}$. Overexpression of anti-apoptotic proteins such as BCL-2 and Bcl-xl were linked to chemoresistance. Pro-apoptotic such as BAX and anti-apoptotic proteins were reported as the targets of JAK2/STAT signaling in various tumor such as leukemia and lymphoma ${ }^{18,21}$. Hence we evaluated the role of anlotinib in regulating those proteins of LSCs. As shown in the figure, upregulation of Bax and inversely downregulation of Bcl-2 and Bcl-xl in Kasumi-1 and KG-1a were observed in anlotinib-treated group(Fig.4A,B) $₫ T$ Thereafter, we further evaluated the role of anlotinib in modulating chemosensitivity by combining with idarubicin (IDA). As a result, anlotinib could significantly enhance the sensitivity of Kasumi-1 and KG-1a to IDA (Fig.4C,D).

Table 1 Clinical characteristics of AML patients were summarized 


\begin{tabular}{|c|c|c|c|c|c|c|c|}
\hline Patient & Gender & Age & FAB & $\begin{array}{l}\text { WBC } \\
\left(10^{\wedge} 9 / \mathrm{L}\right)\end{array}$ & $\begin{array}{l}\text { Blasts } \\
(\%)\end{array}$ & cytogenetics & $\begin{array}{l}\text { Molecular } \\
\text { Mutations }\end{array}$ \\
\hline 1 & $M$ & 39 & M1 & 102.03 & 90.7 & $46 \llbracket X Y$ & ASXL1,IDH1,TET2 \\
\hline 2 & $\mathrm{~F}$ & 34 & M5b & 74 & 10.1 & $46, X X$ & NPM1,FLT3-ITD \\
\hline 3 & $M$ & 51 & M2 & 122 & 81.5 & $46 \llbracket X Y$ & CEBPA,TET2,ASXL1 \\
\hline 4 & $M$ & 45 & M5 & 190.3 & 79.5 & $46 \llbracket X Y$ & FLT3-ITD, NPM1 \\
\hline 5 & $M$ & 38 & M2a & 29.3 & 46 & $46, X Y$ & NRAS,PHF6,TET2,TP53 \\
\hline 6 & $M$ & 40 & M2 & 10.75 & 69.3 & $46, X Y$ & CEBPA,TAD1,bZIP \\
\hline 7 & $\mathrm{~F}$ & 39 & M5b & 48.9 & 59.5 & $\begin{array}{l}\text { 46,XX,t (9: } \\
17 ; 22) \text { (q34, q21, } \\
\text { q11) }\end{array}$ & MLL-AF9 \\
\hline 8 & M & 56 & $\mathrm{M} 2 \mathrm{~b}$ & 44.4 & 21.7 & $\begin{array}{l}45 X, Y, t(8 ; 21) \\
\text { (q22;q22) }\end{array}$ & CEBPA \\
\hline 9 & $M$ & 16 & M2 & 41.7 & 81 & $46, X Y$ & TET2, CEBPA \\
\hline
\end{tabular}

\section{Discussion}

The current study demonstrated anlotinib displayed the effective anti-LSC activity which might associated with the inhibition of JAK2-STAT3/5 signaling. Additionally, anlotinib could significantly enhanced the sensitivity of LSCs to IDA by downregulating Bcl-2. Our results provide a strong scientific rationale and impetus for application of anlotinib in AML.

As reported by Cook, A. M. et al , JAK2-STAT signaling overactivation caused by various cytokines or growth factors or mutation of receptor tyrosine kinases such as c-kit, FLT3 or PDGFR was associated with self-renewal of $\mathrm{LSC}^{19}$. Anlotinib could not only directly induce the apoptosis of LSC but also could inhibit the regeneration of LSC in vivo. Compared with the JAK2 inhibitor, anlotinib showed the mechanistic advantages by the inhibition of JAK2-STAT3/STAT5 activation and STAT3 and STAT5 expression. Such advantages might be associated with multiple target inhibition by anlotinib such as c-kit and PDGFR which could activate other oncogenic signaling including Ras/MAPK囚PI3K/AKT and NF-KB signaling besides JAK2 signaling ${ }^{2}$. However, further study are warranted to investigate the detailed mechanism. Additionally, hematopoiesis toxicity was reported as one of main adverse effects of JAK2 inhibitor in clinical practice ${ }^{1}$, which limited their widely application. However, both clinical data in non-small cell lung cancer ${ }^{13}$ and our laboratory data showed that anlotinib spared no adverse effect on normal mononuclear cells.

IDA combined with cytarabine regimen is the frontline therapy for AML patients ${ }^{3}$. BCL-2 could interact with pro-apoptotic $\mathrm{BH} 3$ only proteins such as $\mathrm{Bax}, \mathrm{BIM}, \mathrm{BID}$ and subsequently blockade intrinsic apoptotic 
pathway ${ }^{15}$. Bcl-2 upregulation in leukemia cells contributed predominantly to the resistance of IDA or cytarabine in AML, which is one of main targets of JAK2-STAT3/5 pathway ${ }^{20}$. Inhibition of JAK signaling could downregulate the expression of Bcl-2 was demonstrated in lymphoma and pulmonary fibrosis ${ }^{8,21}$. Consistent with the inhibition of JAK2-STAT3/5, anlotinib could also significantly decrease Bcl-2 expression and upregulate Bax expression in LSC-like cell lines. Combinatory experiments suggested anlotinib could enhance the sensitivity of LSCs to IDA.

In conclusion, our preclinical findings provide the evidence for clinical implementation of anlotinib as a novel approach to eliminating LSCs and further displayed the synergistic potential of combination of anlotinib with IDA. Considering the limited toxicity of hematopoiesis and its synergic effect with IDA, anlotibib combined with IA regimen might be a promising strategy to deeper clearance of LSCs without causing further myelosuppression.

\section{Declarations}

Fundings: The work was supported by National Natural Science Foundation of China (Grant No.81570156, 81770126, 81800163 and 81770161).

Conflicts of interest: The authors declare no competing financial interests

Ethics approval: All samples were collected after patients were informed consent and were carried out in accordance with the Declaration of Helsinki, and approved by the Ethics Review Board of First Affiliated Hospital of Xiamen University.

Consent to participate: All samples were collected after patients were informed consent.

Consent for publication: Not applicable.

\section{Availability of data and material:}

All authors ensure that all data and materials as well as software application support our published data and comply with field standards.

\section{Authors' Contribution}

Bing Xu, Guowei Li, Yirong Jiang: performed study concept and design and review and revision of the paper; Long Liu and Zhifeng Li: performed development of methodology and writing, review and revision of the paper; Yuelong Jiang, Liying Feng and Qiuling Chen: provided acquisition of experimental data, Yuelong Jiang: performed analysis and interpretation of data, and statistical analysis; Zhifeng Li, Long Liu and Zhijuan Lin: provided the patients samples. All authors read and approved the final paper.

\section{References}


1. Ajayi, S.(2018).Ruxolitinib. Recent Results Cancer Res. 212: p. 119-132.

2. Berenstein, R.(2015). Class III Receptor Tyrosine Kinases in Acute Leukemia - Biological Functions and Modern Laboratory Analysis. Biomark Insights. 10(Suppl 3): p. 1-14.

3. Carter, J.L.(2020). Targeting multiple signaling pathways: the new approach to acute myeloid leukemia therapy. Signal Transduct Target Ther. 5(1): p. 288.

4. Cook, A.M.(2014). Role of altered growth factor receptor-mediated JAK2 signaling in growth and maintenance of human acute myeloid leukemia stem cells. Blood. 123(18): p. 2826-37.

5. Deng, M.(2020).Apatinib exhibits cytotoxicity toward leukemia cells by targeting VEGFR2-mediated prosurvival signaling and angiogenesis. Exp Cell Res. 390(1): p. 111934.

6. Ding, Y.(2017). The biomarkers of leukemia stem cells in acute myeloid leukemia. Stem Cell Investig. 4: p. 19.

7. Liang, L.(2019).Autophagy inhibition potentiates the anti-angiogenic property of multikinase inhibitor anlotinib through JAK2/STAT3/VEGFA signaling in non-small cell lung cancer cells. J Exp Clin Cancer Res. 38(1): p. 71.

8. Milara, J.(2018). The JAK2 pathway is activated in idiopathic pulmonary fibrosis. Respir Res. 19(1): p. 24.

9. Saito, Y.(2010).Identification of therapeutic targets for quiescent, chemotherapy-resistant human leukemia stem cells. Sci Transl Med. 2(17): p. 17 ra9.

10. Sami, S.A.(2020).Current and Future Molecular Targets for Acute Myeloid Leukemia Therapy. Curr Treat Options Oncol. 21(1): p. 3.

11. Schepers, K.(2015).Normal and leukemic stem cell niches: insights and therapeutic opportunities. Cell Stem Cell. 16(3): p. 254-67.

12. Seif, F.(2017). The role of JAK-STAT signaling pathway and its regulators in the fate of Thelper cells. Cell Commun Signal. 15(1): p. 23.

13. Shen, G.(2018).Anlotinib: a novel multi-targeting tyrosine kinase inhibitor in clinical development. J Hematol Oncol. 11(1): p. 120.

14. Short, N.J.(2020).Advances in the Treatment of Acute Myeloid Leukemia: New Drugs and New Challenges. Cancer Discov. 10(4): p. 506-525.

15. Singh, R.(2019). Regulation of apoptosis in health and disease: the balancing act of BCL-2 family proteins. Nat Rev Mol Cell Biol.

16. Thomas, D.(2017).Biology and relevance of human acute myeloid leukemia stem cells. Blood. 129(12): p. 1577-1585.

17. Vetrie, D.(2020). The leukaemia stem cell: similarities, differences and clinical prospects in CML and AML. Nat Rev Cancer. 20(3): p. 158-173.

18. Wingelhofer, B.(2018).Implications of STAT3 and STAT5 signaling on gene regulation and chromatin remodeling in hematopoietic cancer. Leukemia. 32(8): p. 1713-1726. 
19. Wojcik, I.(2005).Abnormalities of the P53, MDM2, BCL2 and BAX genes in acute leukemias. Neoplasma. 52(4): p. 318-24.

20. Yogarajah, M.(2018).A concise review of BCL-2 inhibition in acute myeloid leukemia. Expert Rev Hematol. 11(2): p. 145-154.

21. Yumeen, S.(2020). JAK inhibition synergistically potentiates BCL2, BET, HDAC, and proteasome inhibition in advanced CTCL. Blood Adv. 4(10): p. 2213-2226.

\section{Figures}




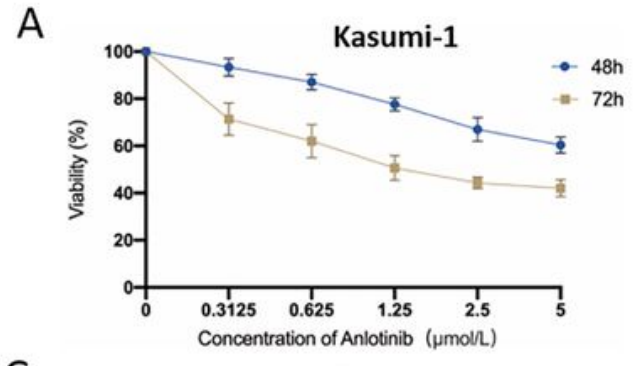

C

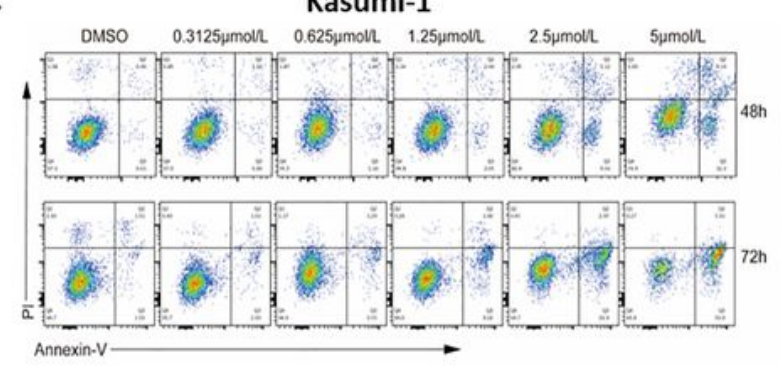

KG-1 $\alpha$

D

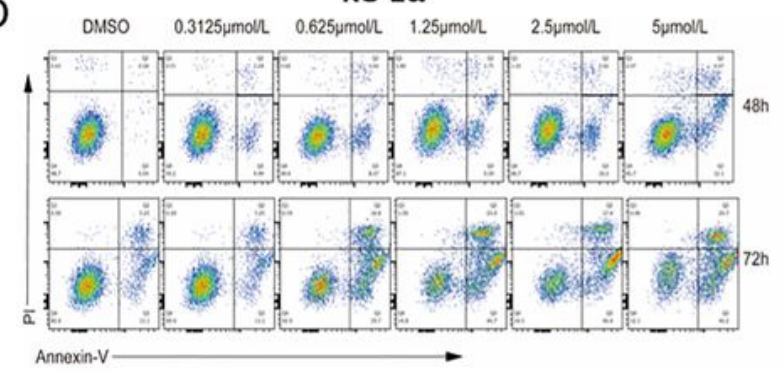

Annexin-V $\longrightarrow$
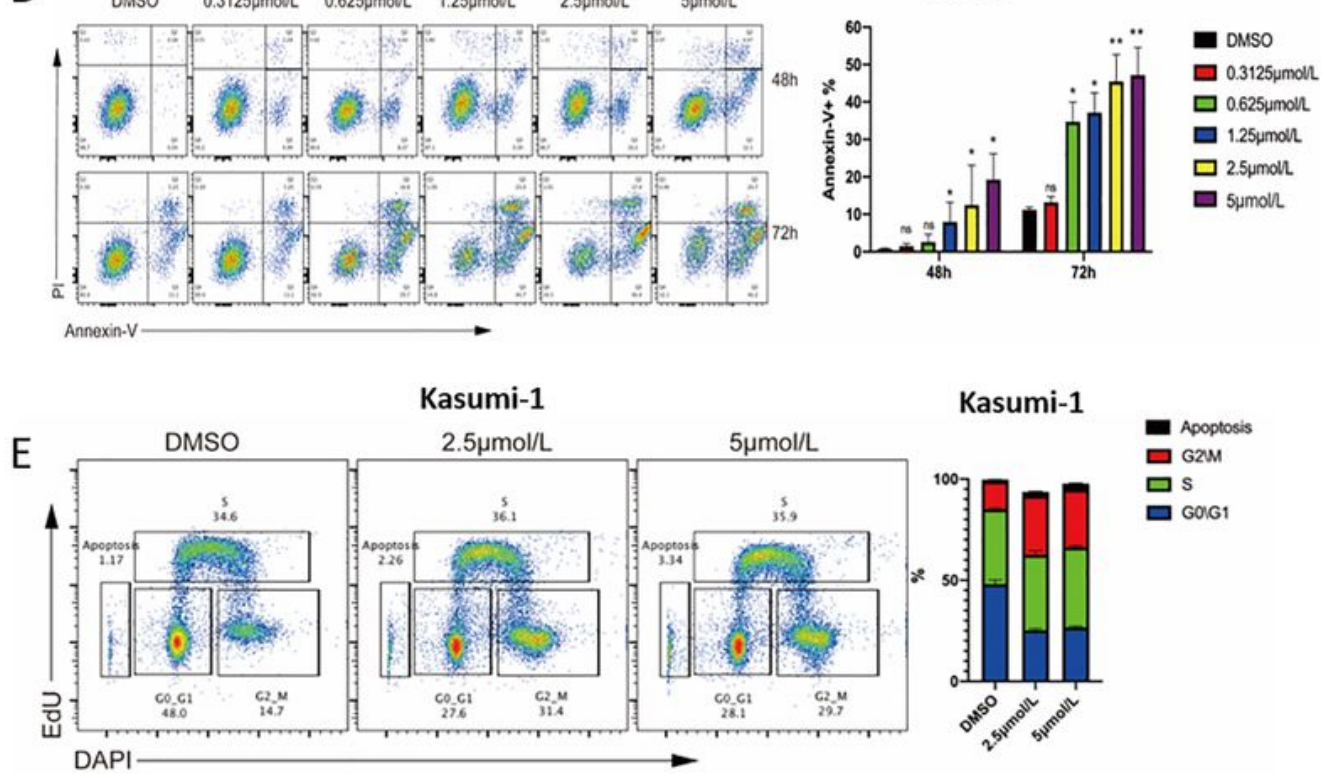

KG-1 $\alpha$

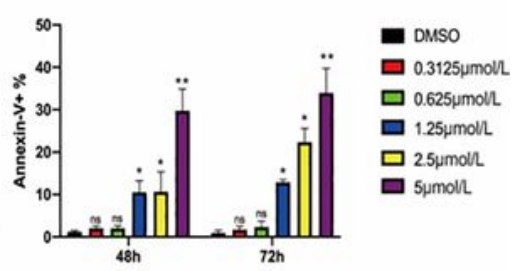

$48 \mathrm{~h}$
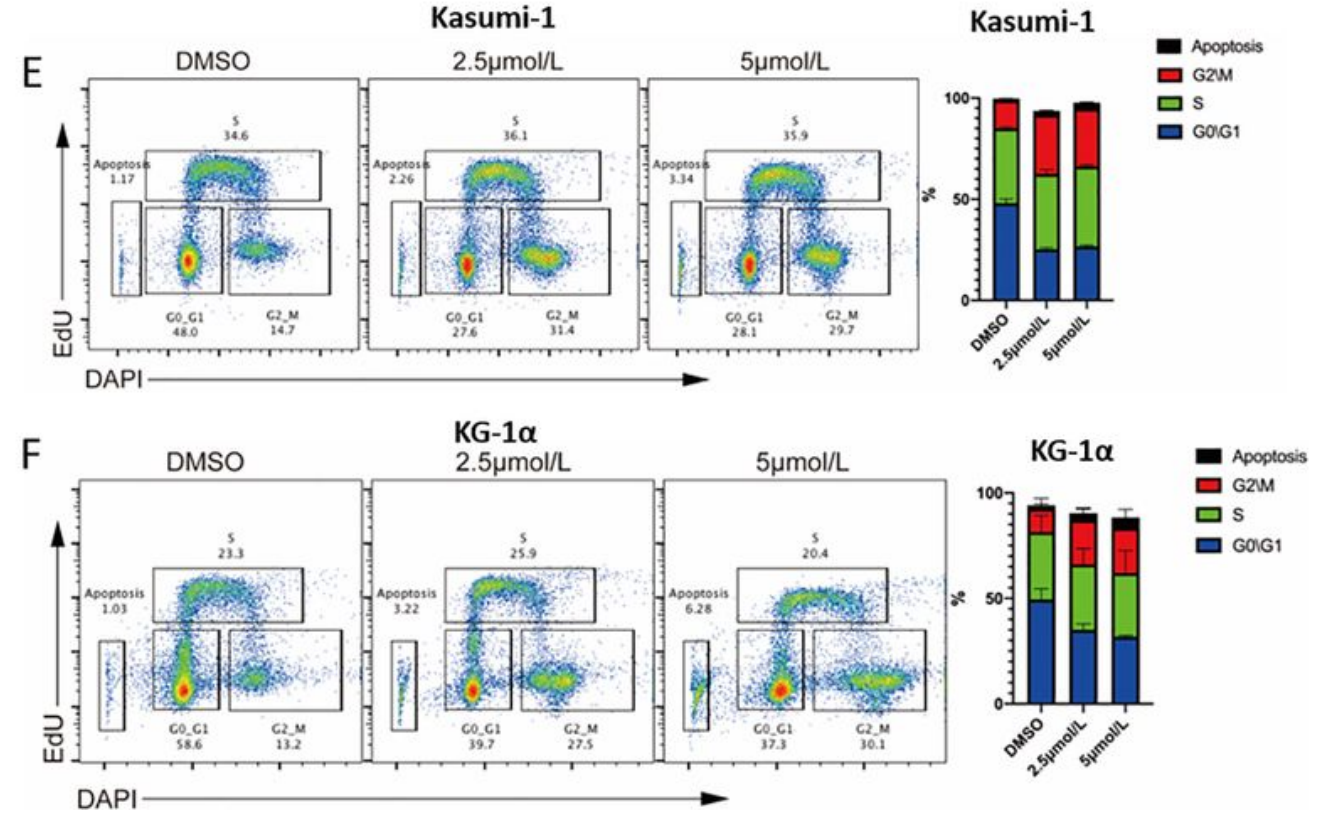

\section{Figure 1}

The anti-LSCs activity of anlotinib in vitro. (A-B) Cell proliferation detected by CCK-8 assay of CD34+CD38- Kasumi-1 cells (A) and KG-1 a cells (B) after treated with the indicated concentrations of anlotinib for $48 \mathrm{~h}$ and 72h. (C-D) The apoptosis of CD34+CD38- Kasumi-1 cells (C) and KG-1a cells (D) after anlotinib treatment with the indicated concentrations of anlotinib for $48 \mathrm{~h}$ and $72 \mathrm{~h}$. (E-F) cell cycle 
detection of CD34+CD38- Kasumi-1 cells (E) and KG-1a cells (F) after anlotinib treatment with the indicated concentrations of anlotinib for $6 h . n=3$ biological replicates ${ }^{*} p<0.05,{ }^{*} p<0.01,{ }^{* *} p<0.001$.

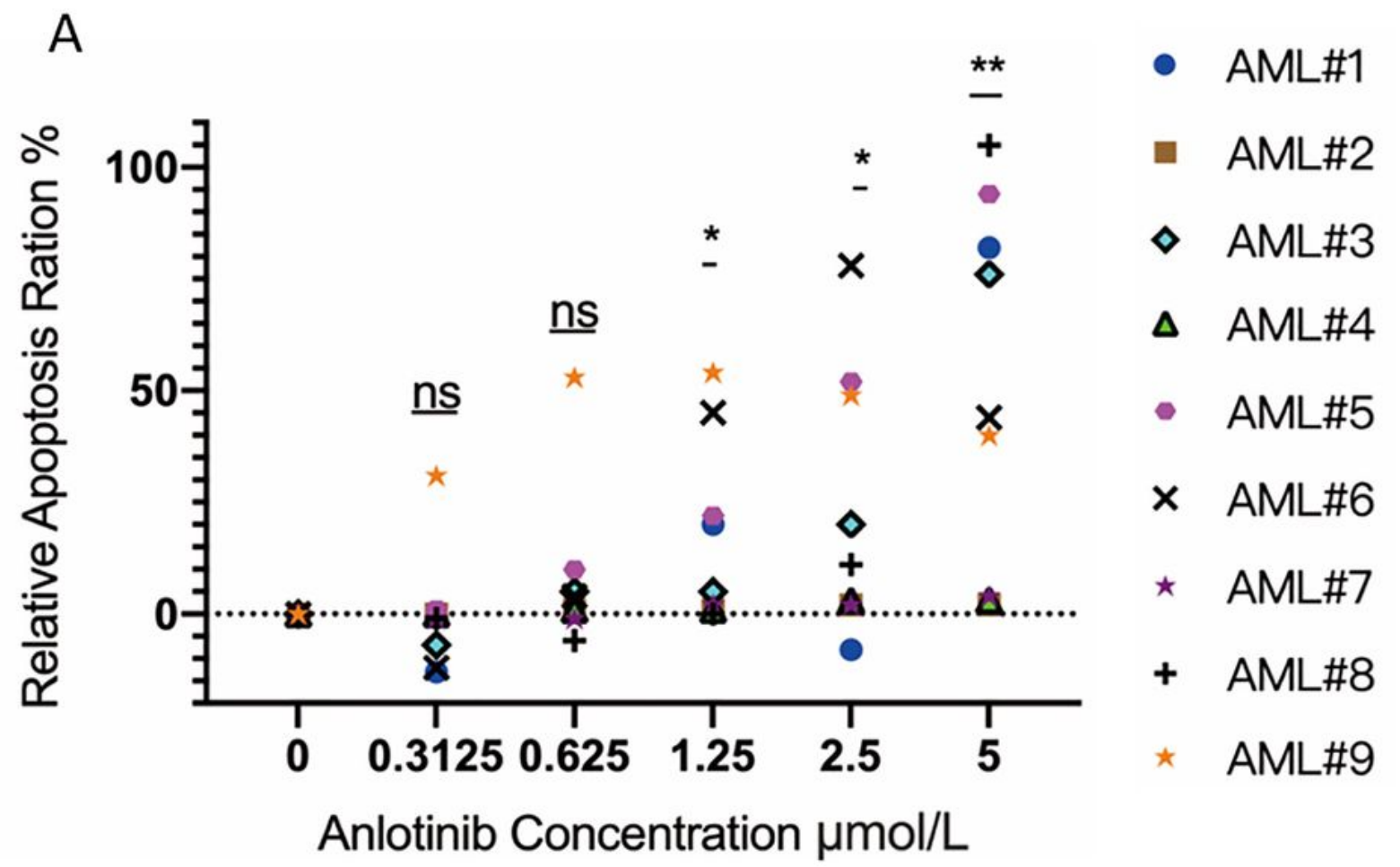

B

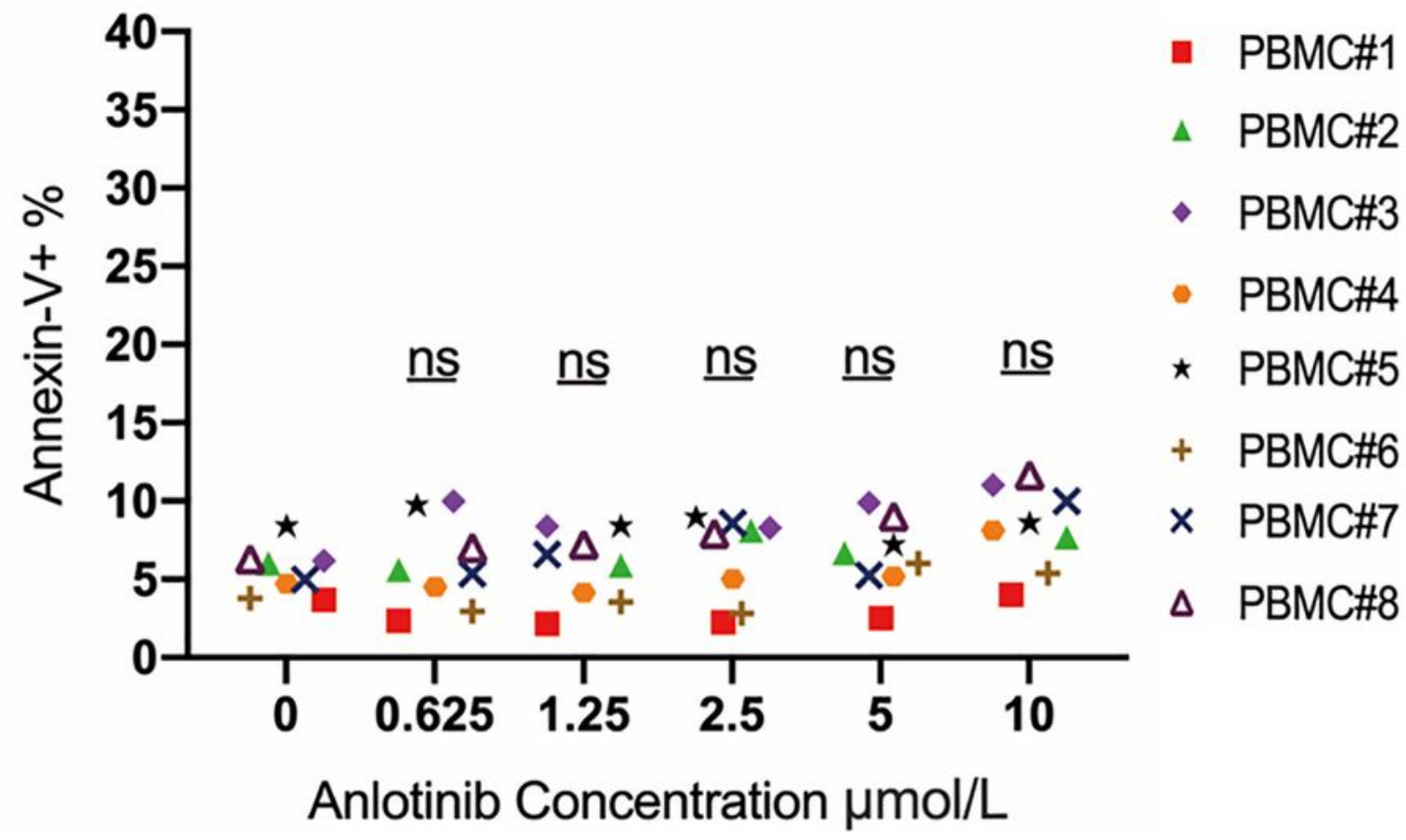

Figure 2

The cytotoxicity of anlotinib on primary CD34+ AML cells and normal leukocytes ex vivo. $\triangle A \backslash T$ The relative apoptosis of CD34+CD38- AML cells after anlotinib treatment with indicated concentration; (B) The 
apoptosis of mononuclear cells from the peripheral blood of healthy donors. ${ }^{\star} p<0.05,{ }^{\star \star} p<0.01, \star \star \star p<$ 0.001 .
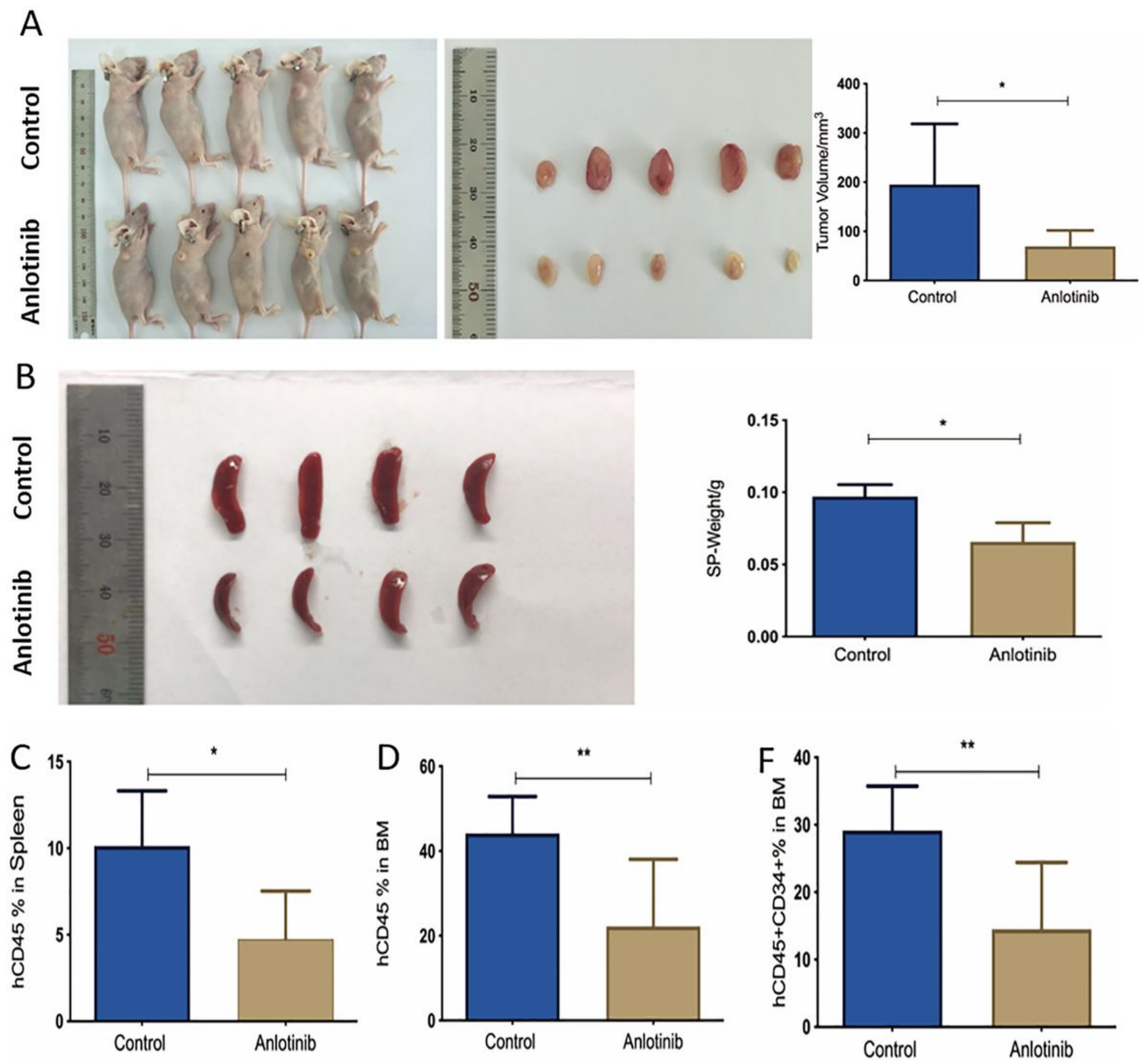

Figure 3

The efficacy of anlotinib in inhibiting LSC-like cells growth and regeneration of LSCs in vivo. (A) The efficacy of anlotinib in the subcutaneous tumorigenic mice model established by Kasumi-1 cells using $B A L B / c$ nude mice ( $n=5$ per group). (B-E)The efficacy of anlotinib in impairing regeneration capacity of LSCs in patient-derived leukemia xenograft mice model. The spleen weight (B) and the percentages of hCD 45+cell in spleen(C) and bone marrow (D) and the percentages of CD45+CD34+cells in bone marrow ( $n=7$ per group). 


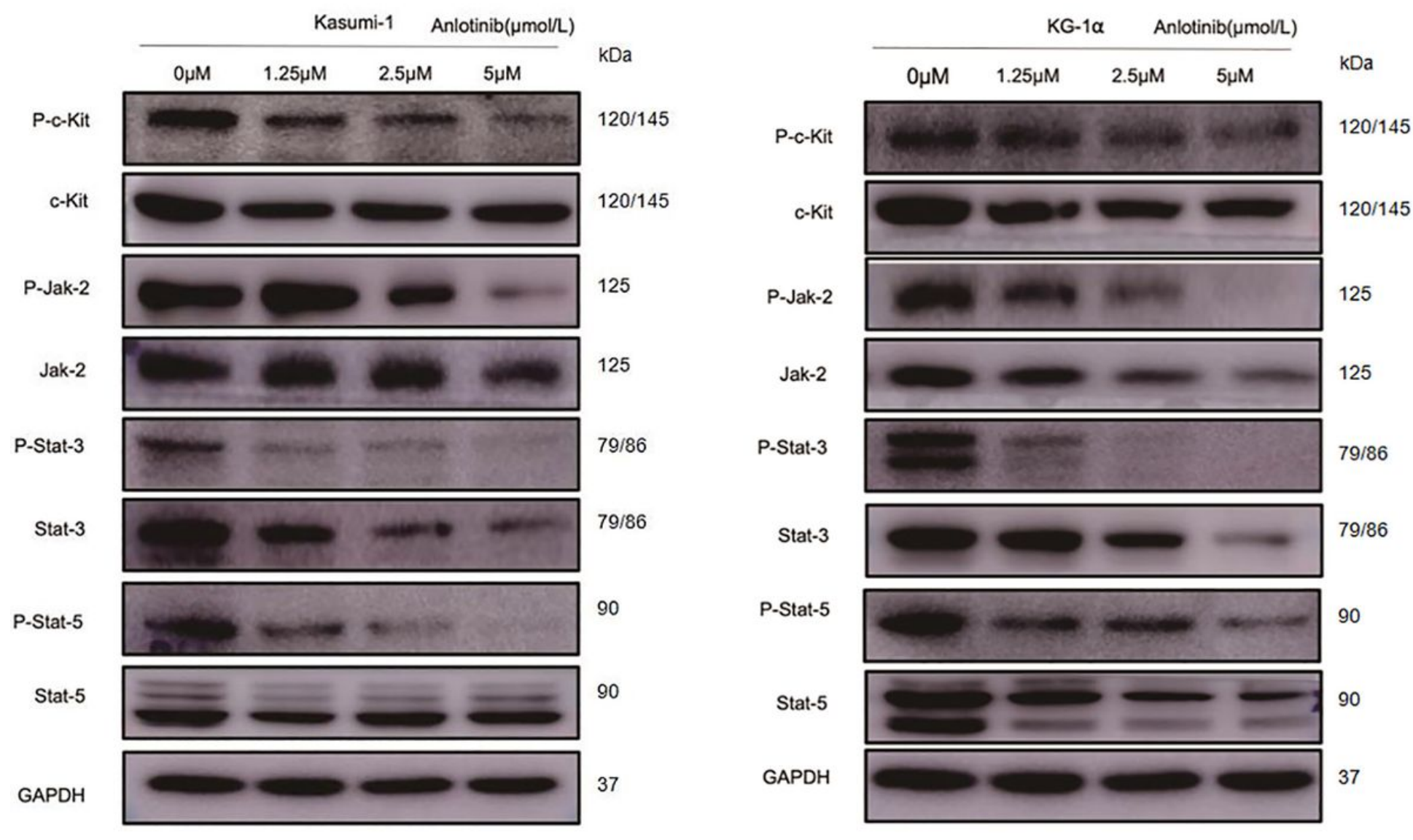

Figure 4

Effects of anlotinib on JAK/STAT signaling in LSC-like cells. (A) Western blot analysis of c-kit, p-c-kit, JAK2, p-JAK2, STAT3, p-STAT3, STAT5, pSTAT5 in KG-1 a cells and Kasumi-1 cells after anlotinib treatment with indicated concentration for $12 \mathrm{~h}$. $\mathrm{n}=3$ biological replicates. 
A

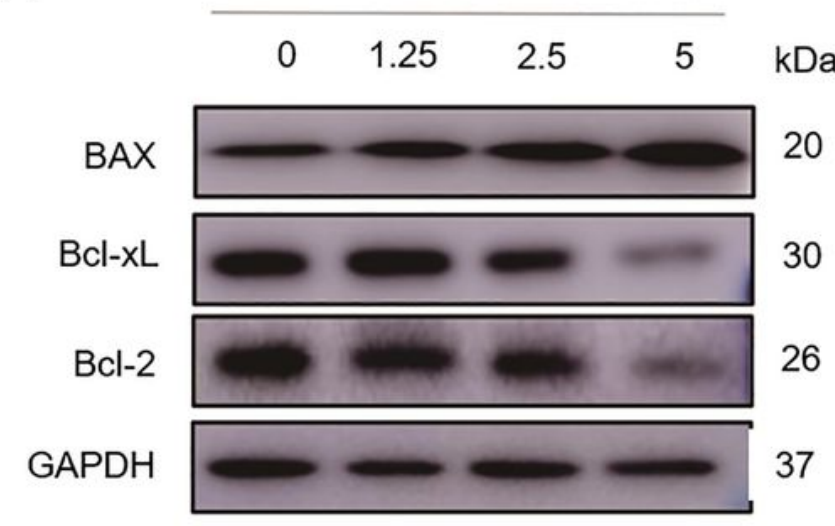

B

KG-1 $\alpha$ Anlotinib ( $\mu M)$

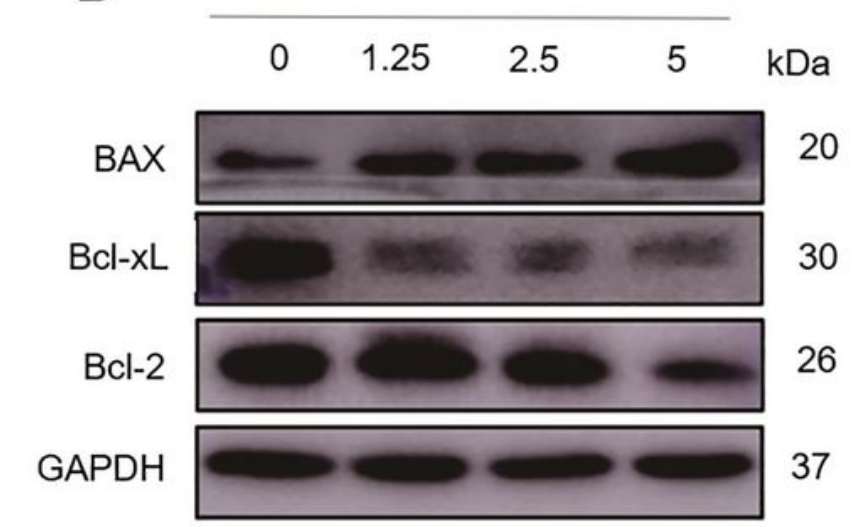

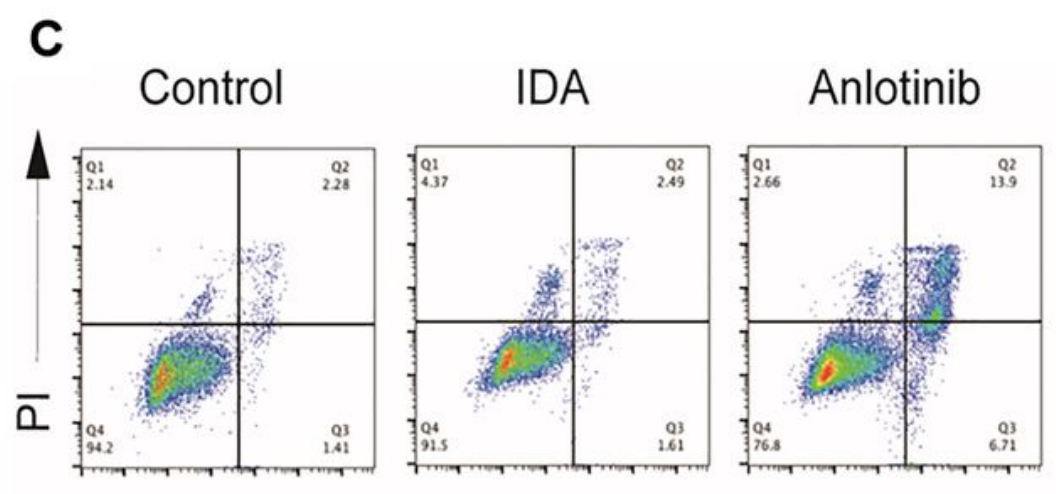
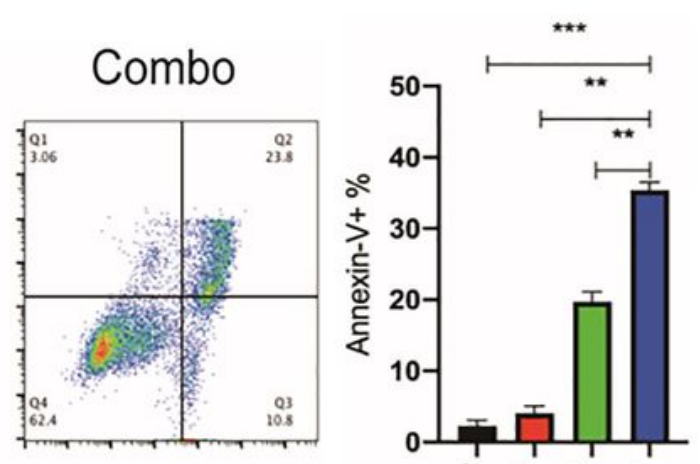

Annexin- $V$
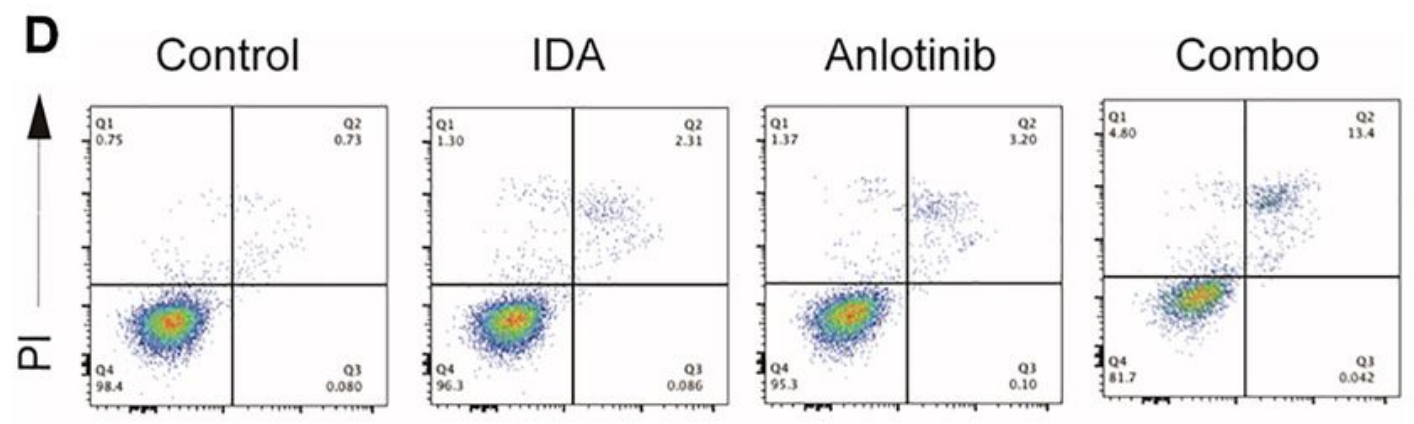

Annexin-V

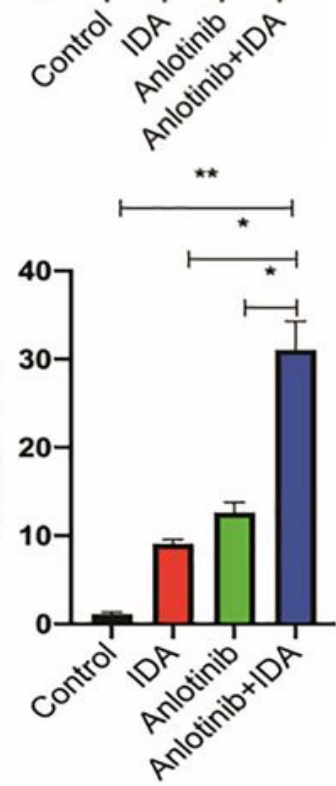

\section{Figure 5}

Effects of anlotinib on apoptotic related proteins in LSC-like cells and its potential in enhancing chemosensitivity of LSC-like cells. (A-B) Western blot analysis of BAX, BCL-2 and BCL-XL in Kasumi-1 cells (A) and KG-1a cells (B) after anlotinib treatment with indicated concentration for 12h.(C-D) The

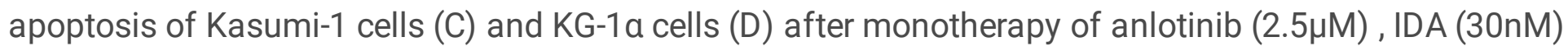


and combinatory therapy of anlotinib $(2.5 \mu \mathrm{M})$ and IDA $(30 \mathrm{nM})$ for $48 \mathrm{~h} . \mathrm{n}=3$ biological replicates ${ }^{*} \mathrm{p}<$ $0.05, * * p<0.01, * \star * p<0.001$.

\section{Supplementary Files}

This is a list of supplementary files associated with this preprint. Click to download.

- graphicabstract.tif 